\title{
Calibrated geodesic foliations of hyperbolic space
}

\author{
Yamile Godoy and Marcos Salvai * \\ FaMAF - CIEM, Ciudad Universitaria, 5000 Córdoba, Argentina \\ ygodoy@famaf.unc.edu.ar, salvai@famaf.unc.edu.ar
}

\begin{abstract}
Let $H$ be the hyperbolic space of dimension $n+1$. A geodesic foliation of $H$ is given by a smooth unit vector field on $H$ all of whose integral curves are geodesics. Each geodesic foliation of $H$ determines an $n$-dimensional submanifold of the $2 n$-dimensional manifold $\mathcal{L}$ of all the oriented geodesics of $H$ (up to orientation preserving reparametrizations). The space $\mathcal{L}$ has a canonical split semi-Riemannian metric induced by the Killing form of the isometry group of $H$. Using a split special Lagrangian calibration, we study the volume maximization problem for a certain class of geometrically distinguished geodesic foliations, whose corresponding submanifolds of $\mathcal{L}$ are space-like.
\end{abstract}

Mathematics Subject Classification: 53C38, 53C12, 53C22, 53C50

Key words and phrases: split special Lagrangian calibration, geodesic foliation, hyperbolic space, space of geodesics

\section{Introduction}

Calibrations are a tool to detect submanifolds of minimum volume in a homology class. One can see their history in the book [13]. They originated with the work [1] of Berger and grew impressively in power in the celebrated paper [8] by Harvey and Lawson of 1982. Dadok, Harvey and Morgan [2, 12] obtained new important examples in 1983. Calibrations provided significant achievements in volume minimization of submanifolds of Euclidean space, and they were also very useful in non-Euclidean settings, for instance in $[14,4,15]$. In 1989 Mealy [11] introduced calibrations on semi-Riemannian manifolds. The split special

\footnotetext{
*Partially supported by CONICET, FONCyT, SECyT (UNC).
} 
Lagrangian calibrations were rediscovered by Warren [17], who applied them to the volume maximization problem of the special Lagrangian submanifolds of a semi-Euclidean space (see also [9]). They also play a central role in the study of optimal transportation [10].

In this article we will use calibrations in connection with geodesic foliations. In this direction, Gluck and Ziller [6] calibrated the Hopf vector fields on the three sphere, which determine the Hopf fibrations, that is, the canonical geodesic foliations of the three sphere. We will deal with the hyperbolic case of arbitary dimension, but instead of calibrating the vector field determining the foliation we will calibrate the foliation itself thought of as the space of its leaves. Since the geodesics of hyperbolic space may be identify with Lorentzian planes in a Minkowski space, the subject of this note is related also with the articles $[14,4]$, dealing with the volume of submanifolds of Grassmannians. In Section 2 we recall basic properties of split special Lagrangian calibrations. The fact that our submanifolds are non-compact makes the definition of volume maximization somehow involved.

Let $H$ be the hyperbolic space of dimension $n+1$. A geodesic foliation of $H$ is given be a smooth unit vector field on $H$ all of whose integral curves are geodesics. Let $\mathcal{L}$ be the space of all the oriented geodesics of $H$ (up to orientation preserving reparametrizations), which is a $2 n$ dimensional manifold and has a canonical split semi-Riemannian metric induced by the Killing form of the isometry group of $H$. A geodesic foliation of $H$ may be identified with an $n$ dimensional submanifold of $\mathcal{L}$. We consider a geometrically distinguished class of geodesic foliations of $H$, which we call t.e.r. geodesic foliations, whose corresponding submanifolds of $\mathcal{L}$ are space-like. This can be found in Section 3.

Now we comment on the contents of the last section. In Theorem 11 we prove that the geodesic foliation $\mathcal{F}_{o}$ orthogonal to a totally geodesic hypersurface of $H$ is volume maximizing among all t.e.r. geodesic foliations. This follows from Theorem 9, where the associated submanifold $\mathcal{M}_{o}$ of $\mathcal{L}$ is proved to be homologically volume maximizing in an open submanifold of $\mathcal{L}$, using a split special Lagrangian calibration.

\section{Calibrations}

Let $N$ be a semi-Riemannian manifold. We will say that an $m$-vector $\xi$ in $T_{q} N$ is space-like if the subspace generated by $\xi$ is space-like (we are not considering the induced indefinite inner product on $\left.\Lambda^{m}\left(T_{q} N\right)\right)$.

We will deal only with split semi-Riemannian manifolds. So, the definitions bellow are given in this case. Specifically, $N$ will be a semi-Riemannian manifold of dimension $2 m$ with signature $(m, m)$. 
Definition 1. Let $N$ be a split semi-Riemannian manifold of dimension $2 m$. A closed $m$-form $\psi$ on $N$ is called a calibration if $\psi_{q}(\xi) \geq \operatorname{vol}_{m}(\xi)$ for any oriented space-like $m$-vector $\xi$ in $T_{q} N$ with $\psi_{q}(\xi)>0$, for all $q \in N$.

Let $\psi$ be a calibration on $N$ and let $M$ be an oriented space-like submanifold of $N$ of dimension $m$. Then $M$ is said to be calibrated by $\psi$ if $\psi_{q}(\xi)=\operatorname{vol}_{m}(\xi)$ for any $m$-vector $\xi$ generating $T_{q} M$ with $\psi_{q}(\xi)>0$, for all $q \in M$.

Definition 2. a) Let $M$ be an oriented $m$-dimensional space-like submanifold of $N$. One says that $M$ is volume maximizing in $N$ if for any open subset $U$ of $M$ with compact closure and smooth border $\partial U$, one has that

$$
\operatorname{vol}_{m}(U) \geq \operatorname{vol}_{m}(V)
$$

for any space-like submanifold $V$ of $N$ of dimension $m$ with compact closure and $\partial V=\partial U$.

b) Moreover, $M$ is said to be homologically volume maximizing in $N$ if in addition $V$ is required to be homologous to $U$.

In this context, uniqueness of $M$ is understood as follows: equality holds in (1) only if $U=V$.

We recall the fundamental theorem of calibrations introduced by Mealy, which is analogous to the corresponding result for Riemannian manifolds in [8].

Theorem 3. [11] Let $N$ be a split semi-Riemannian manifold of dimension $2 m$ and let $\psi$ be a calibration on $N$. If $M$ is an oriented space-like submanifold of $N$ of dimension $m$ which is calibrated by $\psi$, then $M$ is homologically volume maximizing in $N$.

Next we introduce the split special Lagrangian calibration on a split Euclidean space, which appeared first in the work of Mealy [11]. We consider the presentation of this calibration in null coordinates given in [17].

Proposition 4. [17] Let $\mathbb{R}^{n, n}$ be the product $\mathbb{R}^{n} \times \mathbb{R}^{n}$ endowed with the split inner product whose associated square norm is $\|(x, y)\|=\langle x, y\rangle$, where $\langle.,$.$\rangle is the$ canonical inner product on $\mathbb{R}^{n}$. For any $c>0$, the $n$-form

$$
\phi_{c}=\frac{1}{2}\left(c e^{1} \wedge \cdots \wedge e^{n}+\frac{1}{c} f^{1} \wedge \cdots \wedge f^{n}\right)
$$

is a calibration on $\mathbb{R}^{n, n}$ (here $\left\{e^{i} \mid i=1, \ldots, n\right\}$ and $\left\{f^{j} \mid j=1, \ldots, n\right\}$ are the dual of the canonical bases of $\mathbb{R}^{n} \times\{0\}$ and $\{0\} \times \mathbb{R}^{n}$, respectively). Moreover, a space-like $n$-vector $\xi$ is calibrated by $\phi_{c}$ if and only if $\xi$ is special Lagrangian, that is, if

$$
f^{1} \wedge \cdots \wedge f^{n}(\xi)=c^{2} e^{1} \wedge \cdots \wedge e^{n}(\xi) .
$$




\section{Geodesic foliations of hyperbolic space}

\subsection{The space of oriented geodesics of $H$}

Let $H$ be the hyperbolic space of constant sectional curvature -1 and dimension $n+1$. We recall from [16] (see also [3]) some facts about the geometry of the space $\mathcal{L}$ of all complete oriented geodesics of $H$ (up to orientation preserving reparametrizations). It admits a unique differentiable structure of dimension $2 n$ such that the canonical projection $\pi: T^{1} H \rightarrow \mathcal{L}$ is a smooth submersion.

Let $\gamma: \mathbb{R} \rightarrow H$ be a unit speed geodesic and let $\mathcal{J}_{\gamma}$ be the space of all Jacobi vector fields along $\gamma$ which are orthogonal to $\dot{\gamma}$. There exists a well-defined canonical isomorphism

$$
T_{\gamma}: \mathcal{J}_{\gamma} \rightarrow T_{[\gamma]} \mathcal{L}, \quad T_{\gamma}(J)=\left.\frac{d}{d t}\right|_{0}\left[\gamma_{t}\right],
$$

where $\gamma_{t}$ is any variation of $\gamma$ by unit speed geodesics associated with $J$.

Given a tangent vector $X$ to a semi-Riemannian manifold, we denote $\|X\|=$ $\langle X, X\rangle$ and $|X|=\sqrt{|\langle X, X\rangle|}$. Also, given $v \in T H$, we denote by $\gamma_{v}$ the unique geodesic in $H$ with initial velocity $v$.

Let $G$ be the identity component of the isometry group of $H$. The manifold $\mathcal{L}$ is a homogeneous space of $G$ and the Killing form induces on it a semi-Riemannian metric $g$ of signature $(n, n)$. In terms of the isomorphism (2) the square norm of this metric may be written as follows: For $J \in \mathcal{J}_{\gamma}$,

$$
\left\|T_{\gamma}(J)\right\|=|J|^{2}-\left|J^{\prime}\right|^{2},
$$

where $J^{\prime}$ denotes the covariant derivative of $J$ along $\gamma$ (the right hand side in the expression is a constant function, so the left hand side is well defined). For the geometrical meaning of a curve in $(\mathcal{L}, g)$ being space- or time-like see Proposition 4 in $[16]$.

It will be convenient for us to have another presentation of $(\mathcal{L}, g)$. If one considers the unit ball model of $H$, given an oriented geodesic $\gamma$ of $H$, then $\gamma(\infty)$ and $\gamma(-\infty)$ in $S^{n}$ are well defined. Let $\Delta_{n}=\left\{(p, p) \mid p \in S^{n}\right\}$ denote the diagonal in $S^{n} \times S^{n}$. The map

$$
F: \mathcal{L} \rightarrow\left(S^{n} \times S^{n}\right)-\Delta_{n}, \quad F([\gamma])=(\gamma(-\infty), \gamma(\infty))
$$

is a well-defined diffeomorphism.

Given distinct points $p, q \in S^{n}$, let $T_{p, q}$ denote the reflection on $\mathbb{R}^{n+1}$ with respect to the hyperplane orthogonal to $p-q$. Let us consider on $\left(S^{n} \times S^{n}\right)-\Delta_{n}$ the semi-Riemannian metric whose associated norm is

$$
\|(x, y)\|_{(p, q)}=4\left\langle T_{p, q} x, y\right\rangle /|q-p|^{2}
$$


for $x \in p^{\perp}=T_{p} S^{n}, y \in q^{\perp}=T_{q} S^{n}$. Then, if $\mathcal{L}$ is endowed with the metric $g$, the diffeomorphism $F$ given in (4) is an isometry.

\subsection{Geodesic foliations}

A smooth geodesic foliation of $H$ is given by a smooth unit vector field $V$ on $H$ all of whose integral curves, the leaves, are geodesics. In [7] we studied geodesic foliations of the three dimensional hyperbolic space with the approach of Gluck and Warner in [5], where they understand the infinite dimensional manifold of all the great circle foliations of the three sphere. The set $\mathcal{F}$ of all the leaves of a geodesic foliatin of $H$ admits a canonical differentiable structure such that the canonical projection $H \rightarrow \mathcal{F}$ is a smooth submersion and is naturally embedded in $\mathcal{L}$. We will denote by $\mathcal{M}$ the associated submanifold of $\mathcal{L}$, of dimension $n$. The space of oriented geodesics of the three dimensional hyperbolic space admits another canonical split semi-Riemannian metric, apart from that induced by the Killing form. We characterized geometric properties of a geodesic foliation $\mathcal{F}$ in terms of the nondegeneracy of the induced metrics on the corresponding submanifold $\mathcal{M}$ of $\mathcal{L}$.

Now, we consider a new type of geodesic foliations of $H$, for any dimension. Their informal geometrical meaning is that the translational motion of the leaves exceeds their rotational motion (a curve of geodesics in the foliation may be thought of as a motion in $H$ of the initial geodesic), and so we call them t.e.r. geodesic foliations.

Definition 5. A smooth geodesic foliation $\mathcal{F}$ determined by the unit vector field $V$ on $H$ is a t.e.r. geodesic foliation if $\left|\nabla_{v} V\right|<|v|$, for any nonzero tangent vector $v$ of $H$.

Standard examples of t.e.r. geodesic foliations are the foliations which are orthogonal to totally geodesic submanifolds of codimension one of $H$.

Proposition 6. Let $\mathcal{F}$ be a smooth geodesic foliation of $H$. Then $\mathcal{F}$ is a t.e.r. geodesic foliation if and only if the associated submanifold $\mathcal{M}$ of $(\mathcal{L}, g)$ is spacelike.

Proof. Since $\nabla_{V} V=0$, we may suppose that $v$ is orthogonal to $V$. Given a submanifold $\mathcal{M}$ of $\mathcal{L}$ and $[\gamma] \in \mathcal{M}$, any nonzero tangent vector $X$ in $T_{[\gamma]} \mathcal{M}$ corresponds via $T_{\gamma}(2)$ to a Jacobi vector field in $\mathcal{J}_{\gamma}$ associated with a variation of $\gamma$ by unit speed geodesics whose equivalence classes are in $\mathcal{M}$ (see [7]). That is, there exists a smooth curve $c:(-\varepsilon, \varepsilon) \rightarrow H$ with $c^{\prime}(0) \neq 0$ (since $X \neq 0$ ) such that $X=T_{\gamma}(J)$, where $J(s)=\left.\frac{d}{d t}\right|_{0} \gamma_{V(c(t))}(s)$. Now, since $J^{\prime}(0)=\nabla_{J(0)} V$, we have by (3) that

$$
\|X\|=|J(0)|^{2}-\left|J^{\prime}(0)\right|^{2}=\left|c^{\prime}(0)\right|^{2}-\left|\nabla_{J(0)} V\right|^{2} .
$$


Therefore, the equivalence holds.

Let $\varphi_{ \pm}: \mathcal{L} \rightarrow S^{n}$ be the forward (for + ) and backward (for - ) Gauss maps, that is, $\varphi_{ \pm}([\gamma])=\gamma( \pm \infty)$.

Proposition 7. If $\mathcal{F}$ is a t.e.r. geodesic foliation of $H$ and $\mathcal{M}$ is the associated submanifold of $\mathcal{L}$, then $\varphi_{ \pm}: \mathcal{M} \rightarrow S^{n}$ are local diffeomorphisms.

The proof is similar to the proof of the converse in Theorem 4.3 (a) in [7], where Lemma 4.5 in that paper is used (which holds for higher dimensions).

\section{Calibrated geodesic foliations}

In this section we state and prove the main results.

Proposition 8. Let $S$ be a complete totally geodesic submanifold of $H$ of codimension one and let $V$ be a unit vector field normal to $S$. Then

$$
\phi: S \rightarrow \mathcal{L}, \quad \phi(q)=\left[\gamma_{V(q)}\right],
$$

is a space-like submanifold of $(\mathcal{L}, g)$.

Proof. First we observe that $\phi=\pi \circ V$, so $\phi$ is $\operatorname{smooth}(\pi$ is the canonical projection $\left.T^{1} H \rightarrow \mathcal{L}\right)$. Also, it is one to one since $V$ is normal to $S$. Let us see that $(d \phi)_{p}$ is injective for any $p \in S$. Let $w \in T_{p} S$ such that $(d \phi)_{p} w=0$ and let $\alpha:(-\varepsilon, \varepsilon) \rightarrow S$ be a smooth curve with $\alpha^{\prime}(0)=w$. We compute

$$
0=(d \phi)_{p} w=\left.\frac{d}{d t}\right|_{0} \pi \circ V(\alpha(t))=T_{\gamma_{V(p)}}(J),
$$

where $T_{\gamma_{V(p)}}$ is the isomorphism given in (2) and $J(s)=\left.\frac{d}{d t}\right|_{0} \gamma_{V(\alpha(t))}(s)$ is the associated Jacobi vector field in $\mathcal{J}_{\gamma_{V(p)}}$ satisfying $J(0)=w$. Then $J \equiv 0$ and so $w=0$, as desired.

Finally, we check that $\left\|(d \phi)_{p} w\right\|>0$ for any nonzero $w \in T_{p} S$. By (3) and the computation above,

$$
\left\|(d \phi)_{p} w\right\|=|J(0)|^{2}-\left|J^{\prime}(0)\right|^{2}=|w|^{2}-\left|J^{\prime}(0)\right|^{2} .
$$

Since $J^{\prime}(0)=\left.\frac{D}{d t}\right|_{0} V(\alpha(t))$ and $V$ is a parallel vector field along $\alpha$ ( $S$ is a totally geodesic hypersurface), we have that $J^{\prime}(0)=0$ and so $\left\|(d \phi)_{p} w\right\|=|w|^{2}>0$.

Now, we call $\mathcal{M}_{o}$ the image of $\phi$, that is $\mathcal{M}_{o}=\left\{\left[\gamma_{V(q)}\right] \in \mathcal{L} \mid q \in S\right\}$, and denote

$$
\mathcal{L}^{\prime}=\left\{\ell \in \mathcal{L} \mid \ell(\infty) \in \varphi_{+}\left(\mathcal{M}_{o}\right) \text { and } \ell(-\infty) \in \varphi_{-}\left(\mathcal{M}_{o}\right)\right\}
$$

which is an open subset of $\mathcal{L}$.

The following theorem refers to Definition 2 (b). 
Theorem 9. The submanifold $\mathcal{M}_{o}$ is homologically volume maximizing in $\mathcal{L}^{\prime}$ and $\mathcal{M}_{o}$ is unique with this property.

Proof. We work with the Poincaré unit ball model of $H$, included in $\mathbb{R}^{n+1}$. We may suppose without loss of generality that $S=H \cap e_{0}^{\perp}$, where $\left\{e_{0}, \ldots, e_{n}\right\}$ is the canonical basis of $\mathbb{R}^{n+1}$. Since in this model $S$ is an $n$-dimensional ball, we denote it by $B$ (to avoid confusion with the border $S^{n}$ of $H$ ).

By the fundamental theorem of calibrations (Theorem 3) it suffices to find a calibration $\psi$ on $\mathcal{L}^{\prime}$ calibrating $\mathcal{M}_{o}$. In order to define $\psi$ it will be convenient to consider the model of $\mathcal{L}$ given by the isometry $F$ in (4). Via this isometry, $\mathcal{L}^{\prime}$ corresponds to $S_{-}^{n} \times S_{+}^{n}$, where $S_{ \pm}^{n}=\left\{p \in S^{n} \mid \pm p_{0}>0\right\}$ are the upper and lower hemispheres $\left(p_{0}\right.$ is the 0 -th coordinate of $\left.p\right)$. Recall that the open set $S_{-}^{n} \times S_{+}^{n} \subset\left(S^{n} \times S^{n}\right)-\Delta_{n}$ carries the split semi-Riemannian metric (5) induced from the diffeomorphism $F$.

Let $\theta$ be the volume form on the sphere $S^{n}$ with the round metric of constant curvature one. Let $\pi_{i}:\left(S^{n} \times S^{n}\right)-\Delta_{n} \rightarrow S^{n}$ be the projection to the $i$-th factor $(i=1,2)$ and let $\psi$ be the $n$-form on $S_{-}^{n} \times S_{+}^{n}$ defined by

$$
\psi_{(p, q)}=\frac{1}{2}\left(\pi_{1}^{*}\left(\frac{1}{\left|p_{0}\right|^{n}} \theta\right)+\pi_{2}^{*}\left(\frac{1}{\left|q_{0}\right|^{n}} \theta\right)\right) .
$$

Let us see that $\psi$ is a calibration calibrating $F\left(\mathcal{M}_{o}\right)$. Clearly, $\psi$ is closed, since each term is the pull-back of an $n$-form on the $n$-dimensional manifold $S^{n}$.

Now we fix $(p, q)$ in $S_{-}^{n} \times S_{+}^{n}$ and apply Proposition 4 . Let $\left\{e_{1}, \ldots, e_{n}, f_{1}, \ldots, f_{n}\right\}$ be the canonical basis of $\mathbb{R}^{n, n}$ as in that proposition and let $A: \mathbb{R}^{n, n} \rightarrow T_{p} S^{n} \times$ $T_{q} S^{n}$ be the linear isomorphism given by

$$
A\left(e_{i}\right)=\left(v_{i}, 0\right), \quad A\left(f_{j}\right)=\left(0, T_{p, q} v_{j}\right),
$$

where $b=\left\{v_{i} \mid 1 \leq i \leq n\right\}$ is an orthogonal basis of $T_{p} S^{n}$ with $\left|v_{i}\right|=|q-p| / 2$ and $T_{p, q}$ is the reflection defined in the paragraph above (5). Then $A$ is an isometry (the inner product on $T_{p} S^{n} \times T_{q} S^{n}$ is as in (5)). A straightforward computation yields

$$
A^{*} \psi_{(p, q)}=C \phi_{c}
$$

with

$$
C=\frac{|q-p|^{n}}{2^{n}\left|q_{0}\right|^{n / 2}\left|p_{0}\right|^{n / 2}} \quad \text { and } \quad c=\frac{\left|q_{0}\right|^{n / 2}}{\left|p_{0}\right|^{n / 2}} .
$$

Now we see that $C \geq 1$. We compute

$$
|q-p|^{2} \geq\left(q_{0}-p_{0}\right)^{2}=q_{0}^{2}-2 q_{0} p_{0}+p_{0}^{2}=\left|q_{0}\right|^{2}+2\left|q_{0}\right|\left|p_{0}\right|+\left|p_{0}\right|^{2},
$$

since $p_{0}<0$ and $q_{0}>0$. Thus,

$$
|q-p|^{2}-4\left|q_{0}\right|\left|p_{0}\right| \geq\left(\left|q_{0}\right|-\left|p_{0}\right|\right)^{2} \geq 0 .
$$


Hence, $|q-p| \geq 2\left|q_{0}\right|^{1 / 2}\left|p_{0}\right|^{1 / 2}$ and so $C \geq 1$. Consequently, Proposition 4 implies that $\psi$ is a calibration on $S_{-}^{n} \times S_{+}^{n}$.

Next, we verify that the corresponding calibration on $\mathcal{L}^{\prime}$ calibrates $\mathcal{M}_{o}$. We have that

$$
F\left(\mathcal{M}_{o}\right)=\left\{(p, T p) \mid p \in S_{-}^{n}\right\},
$$

where $T: S^{n} \rightarrow S^{n}$ is defined by $T=T_{e_{0},-e_{0}}$, that is, $T\left(p_{0}, p_{1}, \ldots, p_{n}\right)=$ $\left(-p_{0}, p_{1}, \ldots, p_{n},\right)$. Therefore,

$$
T_{(p, T p)} F\left(\mathcal{M}_{o}\right)=\left\{(v, T v) \mid v \in T_{p} S^{n}=p^{\perp}\right\} .
$$

Now, by polarization of (5), for $v_{i} \in b$ as above, the set $\left\{\left(v_{i}, T v_{i}\right) \mid 1 \leq i \leq n\right\}$ is an orthonormal basis of $T_{(p, T p)} F\left(\mathcal{M}_{o}\right)$. We compute

$$
\begin{gathered}
\psi_{(p, T p)}\left(\left(v_{1}, T v_{1}\right) \wedge \cdots \wedge\left(v_{n}, T v_{n}\right)\right)= \\
=\frac{1}{2}\left(\frac{1}{\left|p_{0}\right|^{n}} \theta_{p}\left(v_{1} \wedge \cdots \wedge v_{n}\right)+\frac{1}{\left|-p_{0}\right|^{n}} \theta_{T p}\left(T v_{1} \wedge \cdots \wedge T v_{n}\right)\right) \\
=\frac{1}{2}\left(\frac{1}{\left|p_{0}\right|^{n}}\left(\frac{|p-T p|}{2}\right)^{n}+\frac{1}{\left|-p_{0}\right|^{n}}\left(\frac{|p-T p|}{2}\right)^{n}\right)=1
\end{gathered}
$$

since $T$ is an isometry and $|p-T p|=2\left|p_{0}\right|$.

To conclude, we show the uniqueness. Suppose that $\mathcal{U}$ and $\mathcal{V}$ are as in Definition 2 with $M=\mathcal{M}_{o}$ and $N=\mathcal{L}^{\prime}$. We have

$$
\operatorname{vol}_{n}(\mathcal{V})=\int_{\mathcal{V}} \operatorname{vol}_{n} \leq \int_{\mathcal{V}} i^{*} \psi=\int_{\mathcal{U}} i^{*} \psi=\operatorname{vol}_{n}(\mathcal{U})
$$

(here $i$ is the inclusion; the inequality holds since $\psi$ is a calibration, the last and next to last equalities hold since $\mathcal{U}$ is calibrated by $\psi$ and $\mathcal{U}$ and $\mathcal{V}$ are homologous, respectively). If $\operatorname{vol}_{n}(\mathcal{V})=\operatorname{vol}_{n}(\mathcal{U})$, then $\operatorname{vol}_{n}=i^{*} \psi$ on $\mathcal{V}$ and this is only possible if $\mathcal{V}=\mathcal{U}$ since (6) yields that $C>1$ outside $\mathcal{M}_{o}$.

In the proof of the next theorem we will need the following elementary topological lemma. We give the proof for the sake of completeness.

Lemma 10. Let $B$ be as above the open ball of radius one centered at the origin of $\mathbb{R}^{n+1}$ and let $h: B \rightarrow B$ be a local homeomorphism such that $h$ coincides with the identity on the complement of a closed ball contained in $B$. Then $h$ is a homeomorphism.

Proof. Notice that $h$ extends continuously to the compact ball $\bar{B}$. The map $h$ is onto $B$ since otherwise the sphere $S^{n}$ would be a deformation retract of $B$, a contradiction. Now we check that $h$ is one to one. Since $B$ is simply connected, it suffices to see that $h$ is a covering map. For $q \in B$, the preimage of $\{q\}$ consists of a finite number of points $\left\{p_{1}, \ldots, p_{m}\right\}$ (it can not contain an accumulation 
point, since $h$ is locally one to one). Since $h$ is a local homeomorphism, there exist open neighborhoods $U_{1}, \ldots, U_{m}$ and $V$ of $p_{1}, \ldots, p_{m}$ and $q$, respectively, such that $\left.h\right|_{U_{i}}: U_{i} \rightarrow V$ are homeomorphisms. It remains to show that there exists an open neighborhood $W \subset V$ of $q$ satisfying that $h^{-1}(W) \subset U_{1} \cup \cdots \cup U_{m}$. For any $k \in \mathbb{N}$, let $B_{k}$ the ball of center $q$ and radius $1 / k$. We can take $W=B_{k_{o}}$ for some $k_{o}$. Indeed, if for each $k$ there exists $x_{k} \in h^{-1}\left(B_{k}\right)$ with $x_{k} \notin U_{1} \cup \cdots \cup U_{m}$, an accumulation point of the sequence $x_{k}$ in $\bar{B}$ should be one of the $p_{i}$ 's or be in the border of $\bar{B}$, which is a contradiction.

Let $\mathcal{F}_{o}$ be the foliation of $H$ determined by the unit vector field $V$ normal to $B$ considered at the beginning of this section. Since $\mathcal{M}_{o}$ is a space-like submanifold of $\mathcal{L}$, by Proposition 6 we know that $\mathcal{F}_{o}$ is a t.e.r. geodesic foliation. By the volume of a t.e.r. geodesic foliation $\mathcal{F}$ we understand the volume of the corresponding submanifold $\mathcal{M}$ of $\mathcal{L}$.

Theorem 11. The foliation $\mathcal{F}_{o}$ is volume maximizing among all t.e.r. geodesic foliations $\mathcal{F}$ of $H$. More precisely, as $\mathcal{M}_{o}$ is not compact, $\mathcal{F}_{o}$ has maximum volume among all t.e.r. geodesic foliations $\mathcal{F}$ of $H$ such that the leaves of $\mathcal{F}$ and $\mathcal{F}_{o}$ intersecting the complement of some compact subset of $B$ coincide.

Remark 12. For the three dimensional hyperbolic space, the space of oriented lines is a Kahler semi-Riemannian manifold $[16,3]$ and $\mathcal{M}_{o}$ is a complex space-like surface. Then the assertion of the theorem can also be proved using the Kahler form as a calibration [11], the semi-Riemannian version of the classical Wirtinger Theorem.

Proof. Let $\mathcal{F}$ be a t.e.r. geodesic foliation of $H$ as in the statement of the theorem and let $\mathcal{M}$ be the corresponding submanifold of $\mathcal{L}$. By Propositions 8 and $6, \mathcal{M}_{o}$ and $\mathcal{M}$ are space-like.

Without loss of generality, we may take the compact subset of $B$ as being a geodesic ball $B_{o}$ centered at 0 . Let $\mathcal{U}$ be the subset of $\mathcal{M}_{o}$ consisting of all the oriented geodesics in $\mathcal{M}_{o}$ intersecting $B$ in $B_{o}$. Then we can write $\mathcal{M}$ as $\mathcal{M}=\left(\mathcal{M}_{o}-\mathcal{U}\right) \cup \mathcal{V}$ for some open space-like submanifold $\mathcal{V}$ of $\mathcal{M}$ of dimension $n$ with compact closure satisfying $\partial \mathcal{V}=\partial \mathcal{U}$.

By Definition 2 (a), we must prove that $\operatorname{vol}_{n}(\mathcal{V}) \leq \operatorname{vol}_{n}(\mathcal{U})$. This inequality will follow from Theorem 9 provided that, according to Definition 2 (b), we verify that $\mathcal{M}$ is contained in $\mathcal{L}^{\prime}$ and that $\mathcal{V}$ is homologous to $\mathcal{U}$.

Let us see first that $\mathcal{M}$ is contained in $\mathcal{L}^{\prime}$, that is, $\varphi_{ \pm}(\mathcal{M}) \subset S_{ \pm}^{n}$. Let $W$ be the unit vector field on $H$ which determines the foliation $\mathcal{F}$. The restriction of $W$ to $B$ coincides with $V$ on the complement of $B_{o}$ and moreover cannot be tangent to $B$ at any point $x$. Otherwise, the geodesic with initial velocity $W(x)$, which remains in $B$ ( $B$ is totally geodesic), would intersect the geodesics of $\mathcal{F}$ intersecting the complement of $B_{o}$, a contradiction. By continuity, $\langle V, W\rangle$ is a 
positive function on $B$. Then the backward and forward Gauss maps carry the geodesics with initial velocities $\left.W\right|_{B}$ to $S_{-}^{n}$ and $S_{+}^{n}$, respectively. Thus, $\mathcal{M} \subset \mathcal{L}^{\prime}$.

Now we prove that $\mathcal{U}$ and $\mathcal{V}$ are homologous in $\mathcal{L}^{\prime}$. In order to do this, it will be convenient to identify $\mathcal{L}^{\prime}$ with the product $B \times B$ and $\mathcal{M}_{o}$ and $\mathcal{M}$ with subsets of $B \times B$, which will turn out to be graphs of certain maps on the ball, and then it will be clear that they are homologous in the product.

Let $p_{ \pm}: B \rightarrow S_{ \pm}^{n}$ be defined by $p_{ \pm}(x)=\gamma_{V(x)}( \pm \infty)$, which is a diffeomorphism. We consider the following diagram

$$
B \times B \stackrel{P}{\longrightarrow} S_{-}^{n} \times S_{+}^{n} \stackrel{F}{\longleftarrow} \mathcal{L}^{\prime},
$$

where $F$ is defined in (4) and $P: B \times B \rightarrow S_{-}^{n} \times S_{+}^{n}$ is given by $P(x, y)=$ $\left(p_{-}(x), p_{+}(y)\right)$. Since both $F$ and $P$ are diffeomorphisms, the map $P^{-1} \circ F$ provides an identification of $\mathcal{L}^{\prime}$ with $B \times B$. We have that $P^{-1} F\left(\mathcal{M}_{o}\right)$ is the graph of the identity on $B$, that is $\{(x, x) \mid x \in B\}$.

Let us see that $P^{-1}(F(\mathcal{M}))$ is the graph of a certain diffeomorphism $f$ of $B$ with the property that $f$ coincides with the identity on the complement of $B_{o}$. Let $q_{ \pm}: B \rightarrow S_{ \pm}^{n}$ be defined by

$$
q_{ \pm}(x)=\gamma_{W(x)}( \pm \infty)
$$

which, by Proposition 7 , is a local diffeomorphism coinciding with $p_{ \pm}$on the complement of $B_{o}$. Then the map $f_{ \pm}:=\left(p_{ \pm}\right)^{-1} \circ q_{ \pm}: B \rightarrow B$ is a local homeomorphism that coincides with the identity on the complement of $B_{o}$. By Lemma $10, f_{ \pm}$is a homeomorphism.

Since $F(\mathcal{M})=\left\{\left(q_{-}(x), q_{+}(x)\right) \mid x \in B\right\}$, then

$$
P^{-1}(F(\mathcal{M}))=\{(x, f(x)) \mid x \in B\},
$$

where $f$ is the homeomorphism of $B$ given by $f=f_{+} \circ\left(f_{-}\right)^{-1}$. Now the subsets $\left\{(x, x) \mid x \in B_{o}\right\}$ and $\left\{(x, f(x)) \mid x \in B_{o}\right\}$ are homologous in $B \times B$ and the proof concludes.

\section{References}

[1] M. Berger, Quelques problèmes de géométrie riemannienne ou deux variations sur les espaces symétriques compacts de rang un, Enseignement Math. 16 (1970), 73-96.

[2] J. Dadok and R. Harvey, Calibrations on $\mathbb{R}^{6}$, Duke Math. J. 50 (1983), $1231-1243$. 
[3] N. Georgiou and B. Guilfoyle, On the space of oriented geodesics of hyperbolic 3-space, Rocky Mountain J. Math. 40 (2010), 1183-1219.

[4] D. Gluck, H. Mackenzie and F. Morgan, Volume-minimizing cycles in Grassmann manifolds, Duke Math. J. 79 (1995), 335-404.

[5] H. Gluck and F. Warner, Great circle fibrations of the three-sphere, Duke Math. J. 50 (1983), 107-132.

[6] H. Gluck and W. Ziller, On the volume of a unit vector field on the three sphere, Comm. Math. Helv. 61 (1986), 177-192.

[7] Y. Godoy and M. Salvai, Global smooth geodesic foliations of the hyperbolic space, arXiv:1411.5701 (2014), submitted to Math. Z. in July 2014.

[8] R. Harvey and H. Lawson, Calibrated geometries, Acta Math. 148 (1982), $47-157$.

[9] _ Split special Lagrangian geometry, Metric and Differential Geometry: The Jeff Cheeger Anniversary Volume (X. Dai and X. Rong, eds.), Springer, 2012, pp. 43-89.

[10] R. Kim, Y. McCann and M. Warren, Pseudo-Riemannian geometry calibrates optimal transportation, Math. Res. Lett. 17 (2010), 1183-1197.

[11] J. Mealy, Volume maximization in semi-Riemannian manifolds, Indiana Univ. Math. J. 40 (1991), 793-814.

[12] F. Morgan, On the singular structure of three-dimensional, area-minimizing surfaces in $\mathbf{R}^{n}$, Trans. Am. Math. Soc. 276 (1983), 137-143.

[13] _ Geometric measure theory. A beginner's guide, Elsevier/Academic Press, Amsterdam, 2009.

[14] F. Morgan and W. Ziller, Calibrated geometries in Grassmann manifolds, Comment. Math. Helv. 64 (1989), 256-268.

[15] M. Salvai, A two point calibration on an $\mathrm{Sp}(1)$ bundle over the three-sphere, J. Differential Geom. 59 (2001), 523-533.

[16] On the geometry of the space of oriented lines of the hyperbolic space, Glasgow Math. J. 49 (2007), 357-366.

[17] M. Warren, Calibrations associated to Monge-Ampère equations, Trans. Am. Math. Soc. 362 (2010), 3947-3962. 